\title{
Design and Experimental Evaluation of a Novel Type Radar Reflector for use in the Marine Environment.
}

\author{
Nikolaos Malachias M.Sc.EE, MIET, Systems Engineering Department, \\ Military Technological College, Muscat, Sultanate of Oman. \\ nikolaos.malachias@mtc.edu.om \\ Ioannis Kakavas M.Sc.SE, MIET, Marine Engineering Department \\ Military Technological College, Muscat, Sultanate of Oman. \\ ioannis.kakavas@mtc.edu.om
}

Said Mohammed Said Al Harthi, Systems Engineering Department, Military Technological College, Muscat, Sultanate of Oman.

\section{8@mtc.edu.om}

Ahmed Said Al Saidi, Systems Engineering Department, Military Technological College, Muscat, Sultanate of Oman. 140051@mtc.edu.om

\section{Synopsis}

The octahedral radar reflector is the one most commonly used today in marine applications, for purposes of increasing the Radar Cross Section (RCS) of small vessels at sea. The use of radar reflectors provides greater radar detection distances, therefore contributes to navigation safety. The octahedral reflector has proven to be relatively inefficient, since its RCS values do not remain fairly constant, but show significant variations resulting to changes of the angle of incidence. RCS variations in certain angles of incidence produce nulls, where the radar detection range is almost zero, thus increasing the probability of collision incidents at sea. The scope of this research is to design and evaluate more RCS efficient radar reflector types compared with the operational performance of the octahedral in X-band, thus contribute to navigation safety in the marine environment. Three new reflector models have been designed and manufactured and their RCS was compared with the octahedral reflector RCS in the Military Technological College (MTC) radar lab (in Xband). For the octahedral reflector's and new models' size and weight, the International Maritime Organization (IMO) Resolution
MSC.164 (78) revised performance standards of radar reflectors have been considered. As a result of the measurements, the reflector model with 30 triangular trihedral elements, with side length of $13 \mathrm{~cm}$, has proven to be the most efficient.

Keywords- Radar Cross Section (RCS), navigation safety, metallic radar reflectors, International Maritime Organization (IMO), Military Technological College (MTC).

\section{Introduction}

RCS is the measure of a target's ability to reflect the incident electromagnetic waves coming from radar signals, towards the direction of a radar receiver. RCS is expressed in square meters $\left(\mathrm{m}^{2}\right)$ or $\mathrm{dBm}^{2}$. Main parameters which affect RCS values, are the radar operating frequency, polarization, as well as the target shape, material and the aspect of the incident electromagnetic waves.

Certain platforms in the marine environment mainly constructed of nonmetallic materials, such as military/naval targets, fishing and sailing boats, require the utilization of efficient metallic radar reflectors, in order to be able to provide enhanced and relatively constant RCS values. By using metallic radar reflectors, these platforms 
are detected in greater distances by radars, when the incidence angle of the radar beam (with respect to azimuth and/or elevation angle) varies. This effect contributes to navigation safety at sea, especially for applications in small fishing and sailing boats. Early detection provides adequate time for appropriate maneuvers at sea and minimizes collision probability. Moreover, by using radar reflectors, naval targets are detected and tracked in appropriate distances, during gunnery exercises. Most of the radar reflectors currently used in the marine environment, are of the type of octahedral corner reflector, shown in Figure 1. However, according to the report (UK Marine Accident Investigation Branch 2007) on the investigation of the loss of the sailing yacht "Ouzo" during the night of 20 to 21 August 2006 in UK, the relatively poor performance of the octahedral corner reflector of the yacht might be one of the major causes of this marine accident.

The purpose of this paper is to present the results of the applied research performed and conducted at the radar laboratory of the MTC in Muscat, Sultanate of Oman, in order to design and evaluate more RCS efficient radar reflector types, compared with the operational performance of the octahedral corner reflector, in the frequency of $9 \mathrm{GHz}$ at the X-band of the electromagnetic spectrum $(8-12 \mathrm{GHz})$. The methodology was based on the radar reflector specifications, of the IMO (IMO RESOLUTION MSC.164 2004).

The IMO radar reflector specifications of the standard octahedral corner reflector, deployed today in real life marine applications (figure 1), were the basis of all the comparisons presented in this research.

\section{The Novel Type Radar Reflector}

\subsection{Radar Cross Section (RCS) - Theory}

According to radar theory (Skolnic 1980, Knott et al 1993) the maximum radar detection range of a target, is given by the following formula:

$$
R_{M a x}=\left[\frac{P_{t} G \sigma A_{e}}{(4 \pi)^{2} S_{\min }}\right]^{1 / 4}
$$

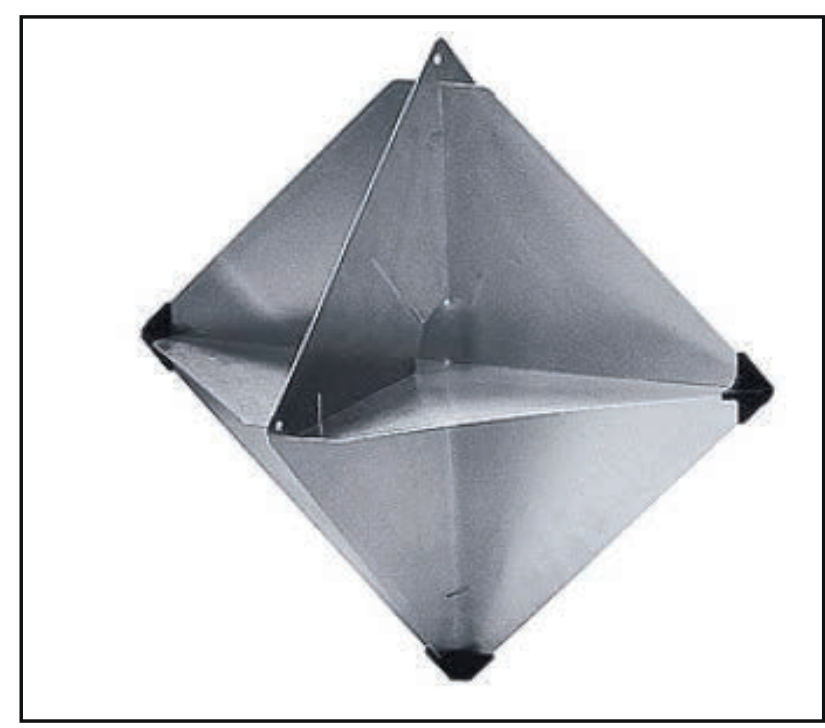

Figure 1: The octahedral corner reflector

Where:

$\mathrm{P}_{\mathrm{t}}$ : Transmitted power

G: Antenna gain

$\sigma$ : Radar cross section

$A_{e}$ : Antenna effective aperture

The RCS of a target (described with the Greek alphabet letter sigma - $\sigma$ ), is expressed in $\mathrm{m}^{2}$ or $\mathrm{dBm}^{2}$ and is defined by the following formula:

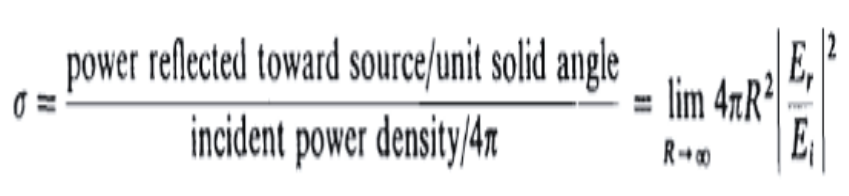

Where:

- R: distance between radar and target

- Er: Reflected field strength at radar

- Ei: strength of incident field at the target

RCS values are affected by the following parameters:

- Radar frequency

- Radar polarization

- Target material

- Target aspect angle

- Target geometry 
RCS $(\sigma)$ of simple shapes that can be obtained by mathematical formulas, are presented in Figure 2 (NAWCWPNS 1999). However, RCS of composite shapes (including marine reflectors) can be only obtained by the following methods:

- Measurement in an appropriate laboratory, with or without an anechoic chamber.

- Software computer simulation.

\subsubsection{IMO specifications of marine radar reflectors}

The revised radar reflector specifications (IMO RESOLUTION MSC.164 2004) are summarized below:

- Radar reflectors should produce reliable detection in practical conditions and perform in both $3 \mathrm{GHz}$ (S-band) and $9 \mathrm{GHz}$ (X-band),

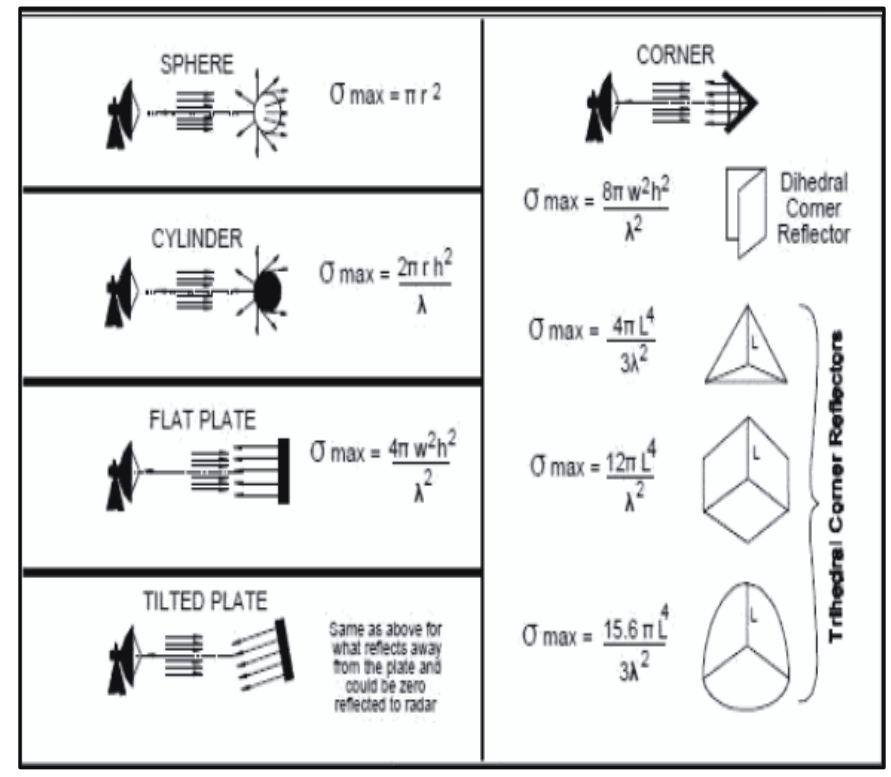

Figure 2: RCS $(\sigma)$, calculation of simple shapes by using mathematical formulas (NAWCWPNS 1999).

- Radar cross sections of at least $7.5 \mathrm{~m}^{2} \mathrm{RCS}$ in the X-band frequency ( $9 \mathrm{GHz}-3 \mathrm{~cm}$ wavelength) and $0.5 \mathrm{~m}^{2} \mathrm{RCS}$ in the S-band $(3 \mathrm{GHz}-10 \mathrm{~cm}$ wavelength).

- This performance level must be maintained over at least $280^{\circ}$ of azimuth and not fall below this level (a "null") over any angle of more than $10^{\circ}$.
- There must be no less than $20^{\circ}$ between nulls.

- For sailing vessels the performance level must be maintained up to at least $20^{\circ}$ of heel, except for multihull vessels which operate with little heel when this minimum should be $10^{\circ}$.

- Physical sizes of reflectors should be minimized and should not exceed $0.05 \mathrm{~m}^{3}$.

\subsubsection{Selection of the reflector models for testing}

Before defining the shape of different reflector models for testing, we selected the most appropriate corner reflector basic element. Next, the reflector models were designed by appropriately combining a number of reflector elements. In order to do so, the relative efficiency of different reflector element was taken into account, in terms of maximum RCS values at the frequencies of interest, as well as RCS variation as a function of the incidence angle.

Three reflector elements were examined, as shown in Figure 3 (Ralph J. F. 2012):

- Triangular trihedral reflector element.

- Circular trihedral reflector element.

- Square trihedral reflector element.

The triangular trihedral reflector element (Figure 4), was chosen for the following reasons:

- It has a fairly good theoretical performance, regarding maximum RCS values in both $3 \mathrm{GHz}$ and $9 \mathrm{GHz}$.

- In particular, at $3 \mathrm{GHz}$ RCS is $0.67 \mathrm{~m}^{2}$ while at $9 \mathrm{GHz}$ is $6 \mathrm{~m}^{2}$. The element side length is $20 \mathrm{~cm}$, which is a typical value for marine reflectors.

- It was expected that RCS values of the reflector models will significantly exceed those values, because they are composed by many triangular trihedral reflector elements. RCS value decreases in a smoother manner, as it happens with the other two reflector elements shown in Figure 3 (Ralph J. F. 2012). 


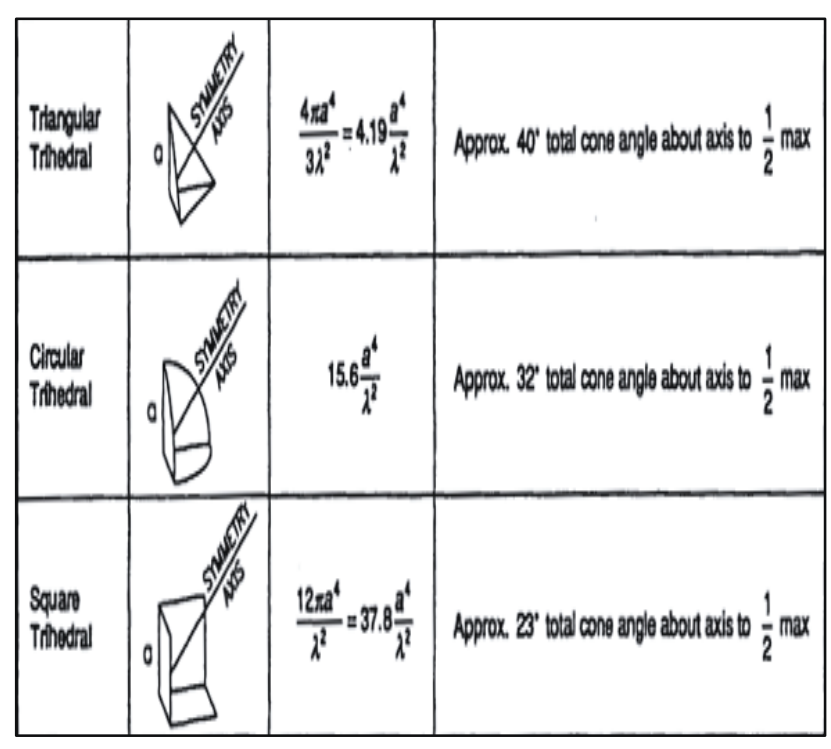

Figure 3: RCS of trihedral shapes (Ralph J. F. 2012)

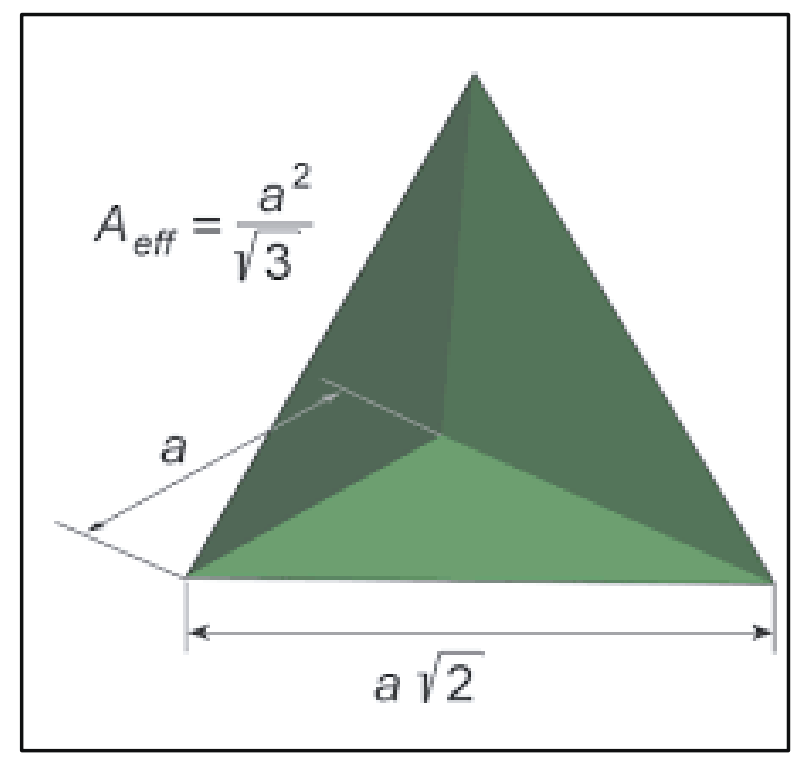

Figure 4: Triangular trihedral reflector element

\subsection{The RCS Measurements}

All the RCS measurements were performed in and conducted with, equipment as well as personnel, of the radar laboratory of MTC, at various elevation angles, for the frequency of 9 $\mathrm{GHz}$ (X-band) as well as various heel angles $\left(0^{0}\right.$, $10^{\circ}$, and $20^{\circ}$ ). The results of the RCS measurements of the octahedral corner reflector, were the basis of comparison with the RCS measurements of the three reflector elements under research. Based on the triangular trihedral reflector element, three more different reflector models were tested in addition to the octahedral corner reflector. The outcome of the measurements was expected to provide satisfactory results regarding the relative efficiency of each reflector model.

At this point, it must be mentioned that in order to obtain high precision RCS data for the reflector which will be finally selected, further measurements should take place in an appropriate anechoic chamber, specially designed for RCS measurements. This advanced measurement facility is not yet available in MTC campus. Moreover, further comparison between the laboratory results and simulation results (i.e. using MATLAB POFACETS or other relevant software), is an item for further research.

By considering that the physical sizes of reflectors should be minimized and should not exceed $0.05 \mathrm{~m}^{3}$ (as per paragraph 4 IMO RESOLUTION MSC.164 2004). The options examined are presented in table 1 and pictured in Figures 5, 6 and 7.

\begin{tabular}{|c|c|c|}
\hline \multicolumn{3}{|c|}{ Reflector Models Used in MTC Research } \\
\hline $\begin{array}{c}\text { Model } \\
\text { number }\end{array}$ & $\begin{array}{c}\text { Number of } \\
\text { elements }\end{array}$ & $\begin{array}{c}\text { Side length } \\
\text { (a) in } \text { cm }\end{array}$ \\
\hline$\# 1$ (octahedral) & 8 & 22 \\
\hline$\# 2$ & 12 & 17 \\
\hline$\# 3$ & 20 & 15 \\
\hline$\# 4$ & 30 & 13 \\
\hline
\end{tabular}

Table 1: Reflector Models used in MTC RCS measurements

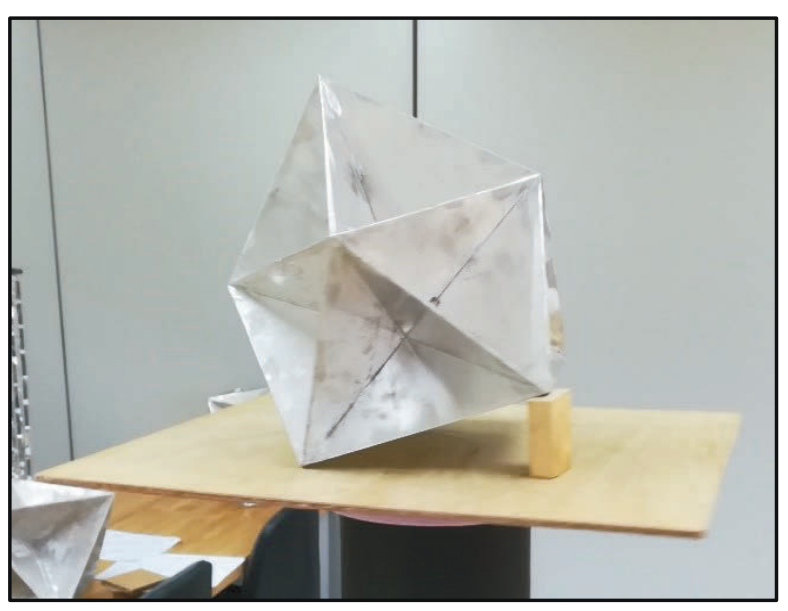

Figure 5: The 12 elements reflector model 


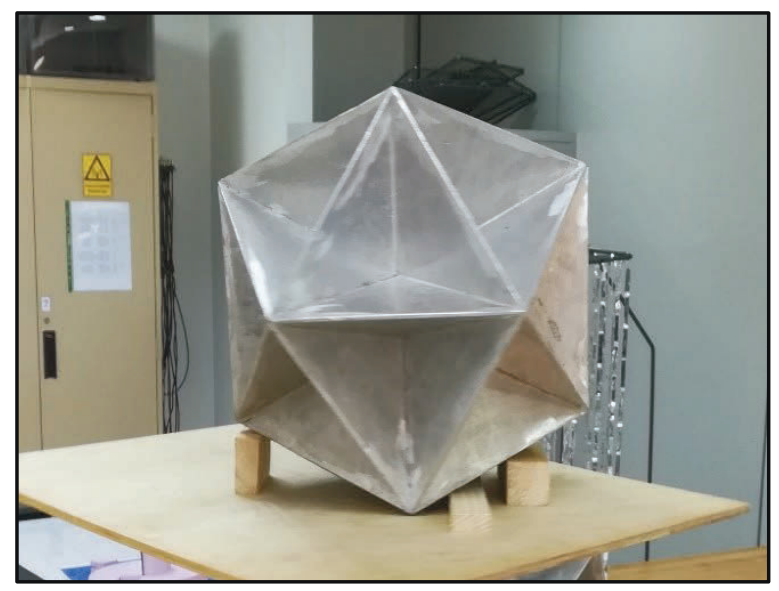

Figure 6: The 20 elements reflector model

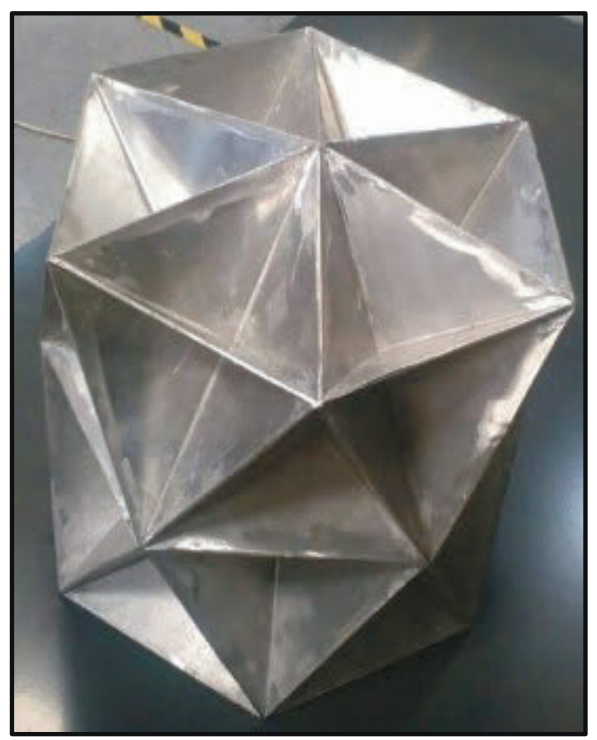

Figure 7: The 30 elements reflector model

The three radar reflector models in Figures 5, 6 and 7, were assembled and produced as prototypes, in the radar laboratory of MTC.

\subsubsection{RCS of the radar reflector models}

Figures 8, 9 and 10 display the comparison of the results of the RCS measurements of the three corner reflector options presented in table 1 and shown in Figures 5, 6 and 7, for the frequency of $9 \mathrm{GHz}$ (X-band), compared with the RCS measurements of the octahedral corner reflector, for the heel angles of $0^{\circ}, 10^{\circ}$ and $20^{\circ}$ respectively. The term heel angle, describes a temporary inclination/deviation around the longitudinal axis of the marine vessel, generally involving motion, such as wind or turning, while roll indicates periodic inclination from side to side, such as wave action. Each corner reflector option, is illustrated in the graphs by a different color as follows:

- Octahedral reflector: Black

- 12 elements reflector: Red

- 20 elements reflector: Green

- 30 elements reflector: Blue

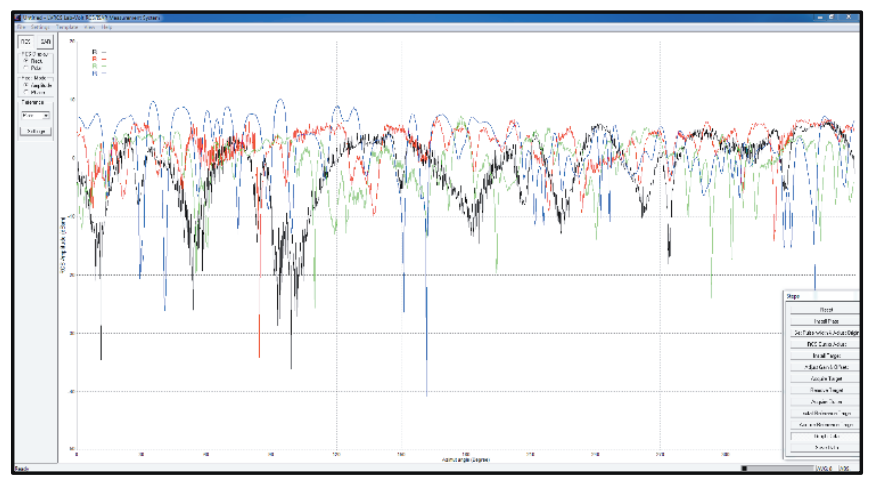

Figure 8: Comparison of the RCS measurements for the heel angle of $0^{0}$

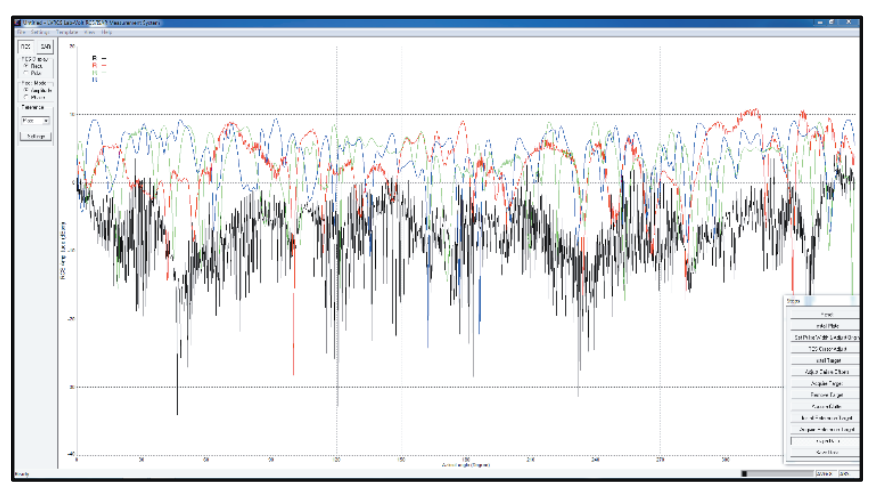

Figure 9: Comparison of the RCS measurements for the heel angle of $10^{\circ}$

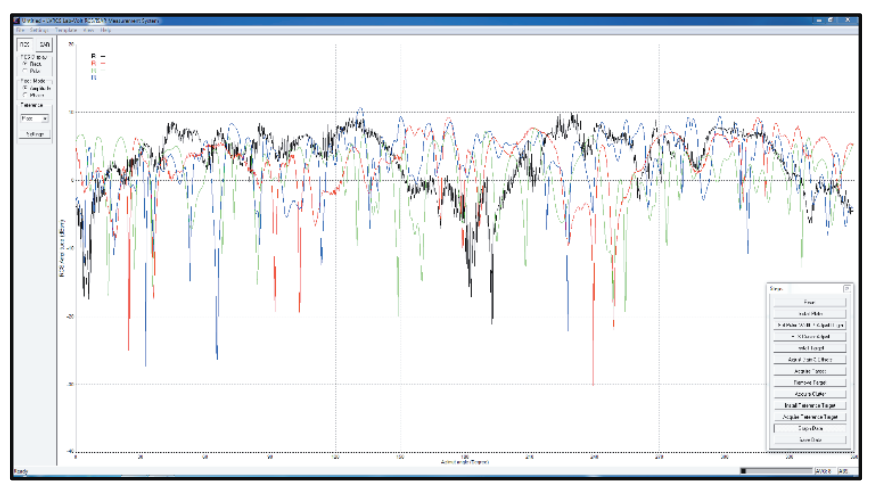

Figure 10: Comparison of the RCS measurements for the heel angle of $20^{\circ}$ 


\subsubsection{Comparison of the RCS measurements.}

The overall performance of the standard octahedral radar reflector, deployed today in real life marine applications (figure 1), was compared with the performance of the other three reflectors at various heel angles.

The following observations are concluded from the RCS measurements:

a. Not a perfect corner radar reflector can be produced for all heel angles, as well as azimuths. There is no silver bullet solution in order to accommodate all safety issues with respect to radar issues in the marine environment.

b. The octahedral radar reflector presents several more nulls among the four reflectors that participated in this research. Its performance can compete with the other three, only at the $20^{\circ}$ heel angle. In this specific angle the standard octahedral radar reflector RCS is considered to be satisfactory enough, as compared with the performance of the three other reflectors that were produced in-house MTC.

c. The most efficient of the four radar reflectors that participated in the research, is the 30 elements reflector.

d. The most efficient radar reflector (RCS vs angle of incidence at $9 \mathrm{GHz}$ ) should be selected, for further evaluation as well as research.

\section{Conclusion}

Under this research more RCS efficient radar reflector types were compared with the operational performance of the octahedral radar reflector in X-band, thus contributed to navigation safety in the marine environment. Three new radar reflector models were designed and manufactured and their RCS was compared with the octahedral reflector RCS. The reflector model with 30 triangular trihedral elements, with side length of $13 \mathrm{~cm}$, has proven to be the most efficient, as compared to the octahedral one.

\section{Acknowledgments}

The authors are extremely grateful to have received full support from the Military Technological College (MTC), in Muscat Sultanate of Oman. MTC offered the required premises, equipment and staff of the radar laboratory to conduct this research. In addition to this, the research has benefited from inputs of a number of MTC students, who contributed to their learning in the RCS study area, by pointed discussions. The project has been approved and funded in 2018, under a scholarship granted by the Research Council of the Sultanate of Oman, for the Faculty Mentored Undergraduate Research Award Program (FURAP).

\section{References}

IMO RESOLUTION MSC.164 (78) - adopted on 17 May 2004.

Knott E., Shaeffer J., Tuley M. 1993. Radar Cross Section, 2nd edition, by. Artech House.

Nathanson F. 1991. Radar Design Principles, 2nd edition by. Mc Graw Hill.

NAWCWPNS-TP8347-Rev.2, April 1999.

Ralph J. F. 2012. Avionics AERO230 (Lecture Notes, University of Liverpool).

Report by QinetiQ: "Performance Investigation of Marine Radar Reflectors on the Market" https://assets.publishing.service.gov.uk/media/5 47c705540f0b6024400008d/Radar_reflectors_r eport.pdf .

Skolnic M. 1980. Introduction to Radar Systems - 2nd edition, Mc Graw Hill.

UK Marine Accident Investigation Branch Report \# 7/2007, April 2007. 